\title{
Giant Inguino-scrotal Hernia: Repair and Management
}

\author{
${ }^{1}$ SM Ikhwan, ${ }^{2}$ AS Saiful, ${ }^{3}$ MI Ibariyah, ${ }^{4}$ Z Zaidi \\ ${ }^{1}$ Department of Surgery School of Medical Sciences Universiti Sains Malaysia Health Campus 16150 Kubang \\ Kerian Kelantan, Malaysia \\ ${ }^{2}$ Department of Surgery Hospital Sultanah Aminah, 8000 Johor Bahru, Johor, Malaysia \\ ${ }^{3}$ Department of Anaesthesiology School of Medical Sciences Universiti Sains Malaysia Health Campus 16150 \\ Kubang Kerian Kelantan, Malaysia \\ ${ }^{4}$ Department of Surgery School of Medical Sciences Universiti Sains Malaysia Health Campus 16150 Kubang \\ Kerian Kelantan, Malaysia
}

\begin{abstract}
An 84-year-old Malay gentleman presented with signs of intestinal obstruction secondary to obstructed right giant inguinoscrotal hernia. He was subjected for emergency inguinal hernia repair with intraoperative findings of multiple loop of dilated bowel with thickened omentum within the sac. Repair of the hernia defect with strengthening of the posterior wall was carried out. Post-operatively the patient was managed in $I C U$ due to development of intra-abdominal hypertension. The presentation and management of giant inguinoscrotal hernia will be further discussed.
\end{abstract}

Keywords: giant inguinoscrotal hernia, abdominal compartment syndrome

\section{Introduction}

Giant inguinal hernias are uncommon in modern surgical practice, but can present a challenging problem to the treating surgeon, as they can lead to potentially fatal complications especially when they presented as obstruction ${ }^{1,4}$.

Giant inguinal hernias are defined as those which extend below the midpoint of inner thigh in the standing position. An older definition puts it as that bigger than the average human head ${ }^{4}$. Such giant hernias are uncommonly encountered in modern surgical practice. Not only they pose special problems in management, but also they reflect the ignorance and neglect of potentially dangerous surgical problems in developing countries such as Malaysia, faith of the people in alternative systems of medicine and to some extent, the inability of modern medical facilities to reach the common man such as in Kelantan.

The main concern for those cases which presented as obstruction is the possible development of intraabdominal hypertension (IAH) and eventually abdominal compartment syndrome (ACS) upon successful reduction of the chronically herniated abdominal contents. The abdominal compartment syndrome is unique as a compartment syndrome in that it affects virtually all organ systems within the body ${ }^{5}$. Organ compromise begins insidiously and at variable intra-abdominal pressures depending on the organ system investigated and the individual's abdominal compliance towards the increasing pressure. Chronically increased intra-abdominal volume, such as that caused by obesity, pregnancy or cirrhotic ascites fluid does not usually cause significant IAH or ACS because the abdominal wall can increase its compliance over time in such situations ${ }^{5,6}$. However, in acute onset such as sudden increase in abdominal pressure or volume which anticipated for example upon successful reduction of hernia contents, may cause significant effect from IAH or ACS as the abdomen unable to compensate such acute rise in pressure.

We are presenting a case of obstructed giant inguinal hernia reaching up to the knees of a patient and its management in Hospital Universiti Sains Malaysia (HUSM).

\section{Case Report}

An 84-year-old Malay gentleman presented to the Emergency Department of HUSM with a history of irreducible right inguino-scrotal swelling for the past 1 week duration. He had history of right inguinal hernia since almost more than 30 years back but did not pay much attention to it as it didn't cause much problem so far. He only took home remedies together with some traditional medicine. It didn't cause much problem to him in relation to his daily activities until it had almost reached his knee. 


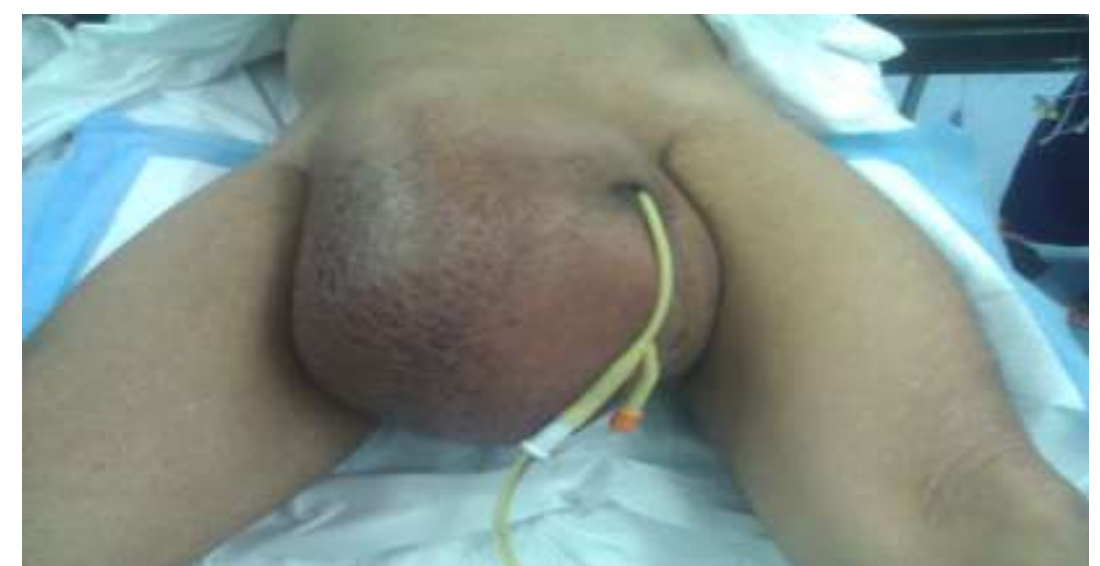

Figure 1: Peri-operative findings of giant right inguinal hernia which was clinically obstructed.

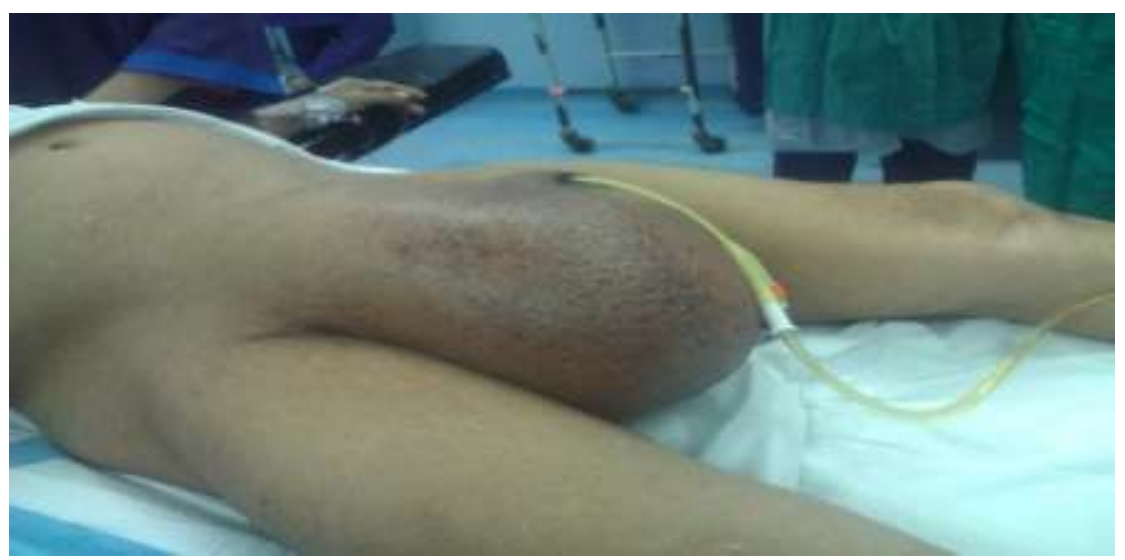

Figure 2

It had been also associated with lower abdominal discomfort and difficulty in passing motion. The abdominal discomfort was initially tolerable, but having increasing in intensity and severity since past few days prior to the admission. He also had progressive abdominal distension and nauseated upon attempt of oral intake. There was no association with fever, respiratory distress or difficulty in micturation.

On examination, he had a huge irreducible right inguino-scrotal swelling which almost reached his knee. The scrotal and penile skins were stretched and slightly erythematous with superficial ulceration on the scrotal bed. The swelling was tensed and tender upon palpation. The right sided testis was unable to be felt and bowel sound can be heard within the swelling. The abdomen was slightly distended but soft and no evidence of peritonitis.

His initial abdominal radiograph showed mildly dilated small bowel. Chest radiograph did not showed any free air under the diaphragm. Blood investigation and blood gases were within normal range.

He was subjected for emergency hernia repair after initial optimization. At operation, the hernia was approached through standard tranverse inguinal incision. Intra-operative findings revealed multiple loops of dilated small and large bowel together with omentum occupying the huge sac.

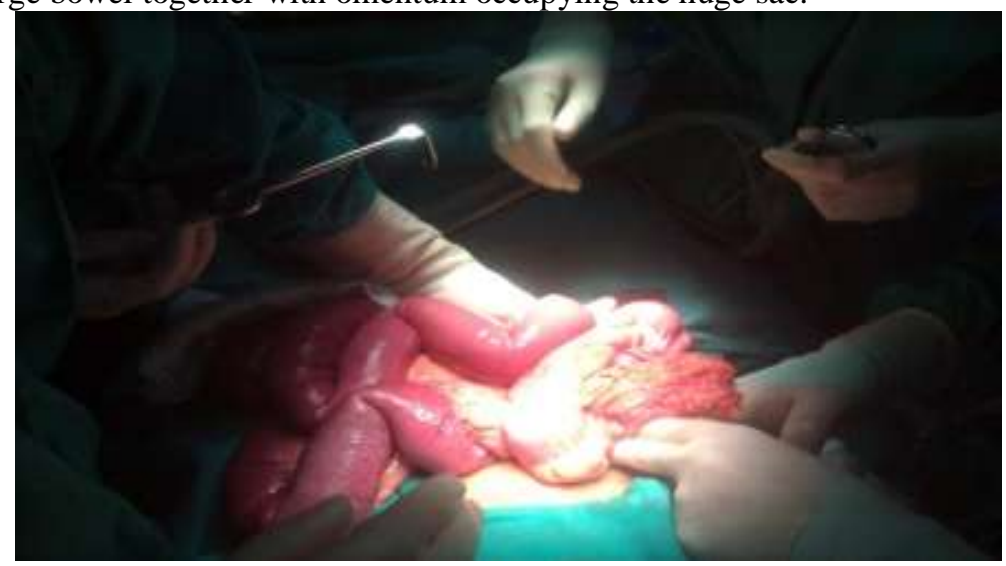

Figure 3: Intraoperative findings. Note marked dilated loops of small bowel. 
The internal inguinal ring was widened in order to reduce such contents. Bowel decompression was carried out through appendicectomy stump. A successful reduction of the contents was achieved without having to proceed for midline laparotomy incision. The internal ring was later repaired and narrowed with Prolene $2 / 0$ suture and the posterior wall was strengthened by dunning overlay suture with Prolene $2 / 0$. No resection of the bowel or any reconstructive procedure of the abdominal wall was done. Post-operatively, he was managed in ICU for further optimization and for close observation of the intra-abdominal pressure.

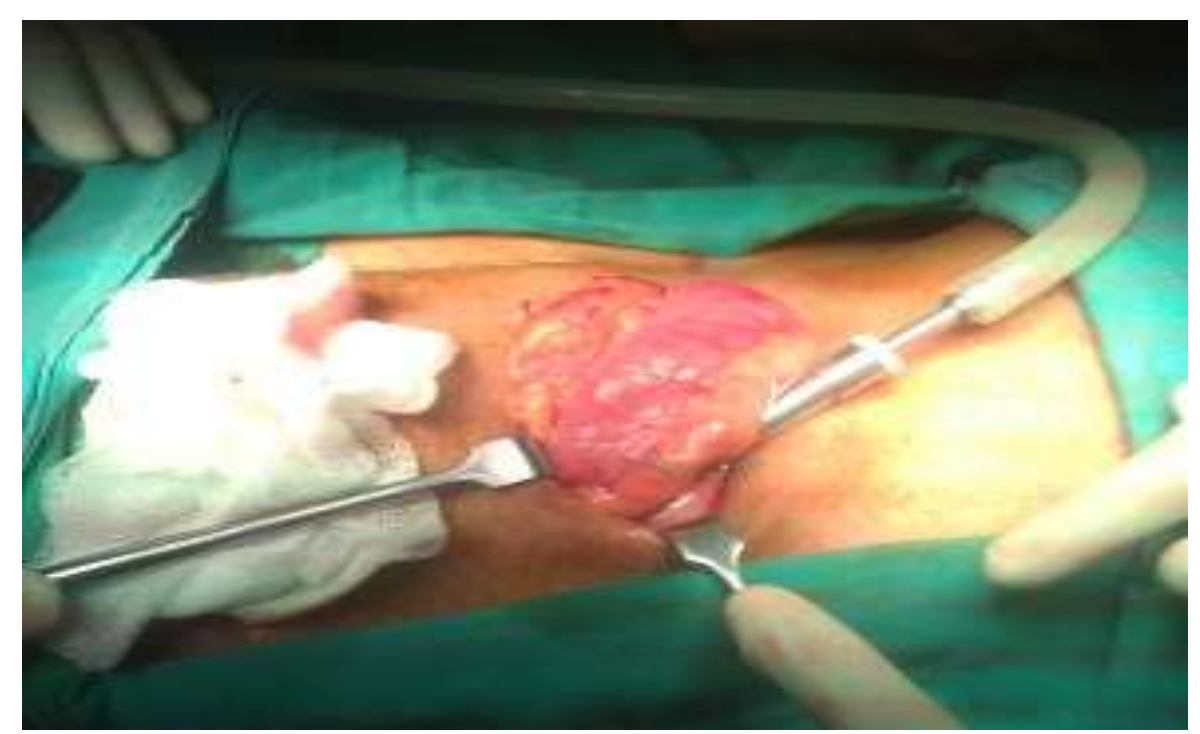

Figure 4: Successful reduction through inguinal incision.

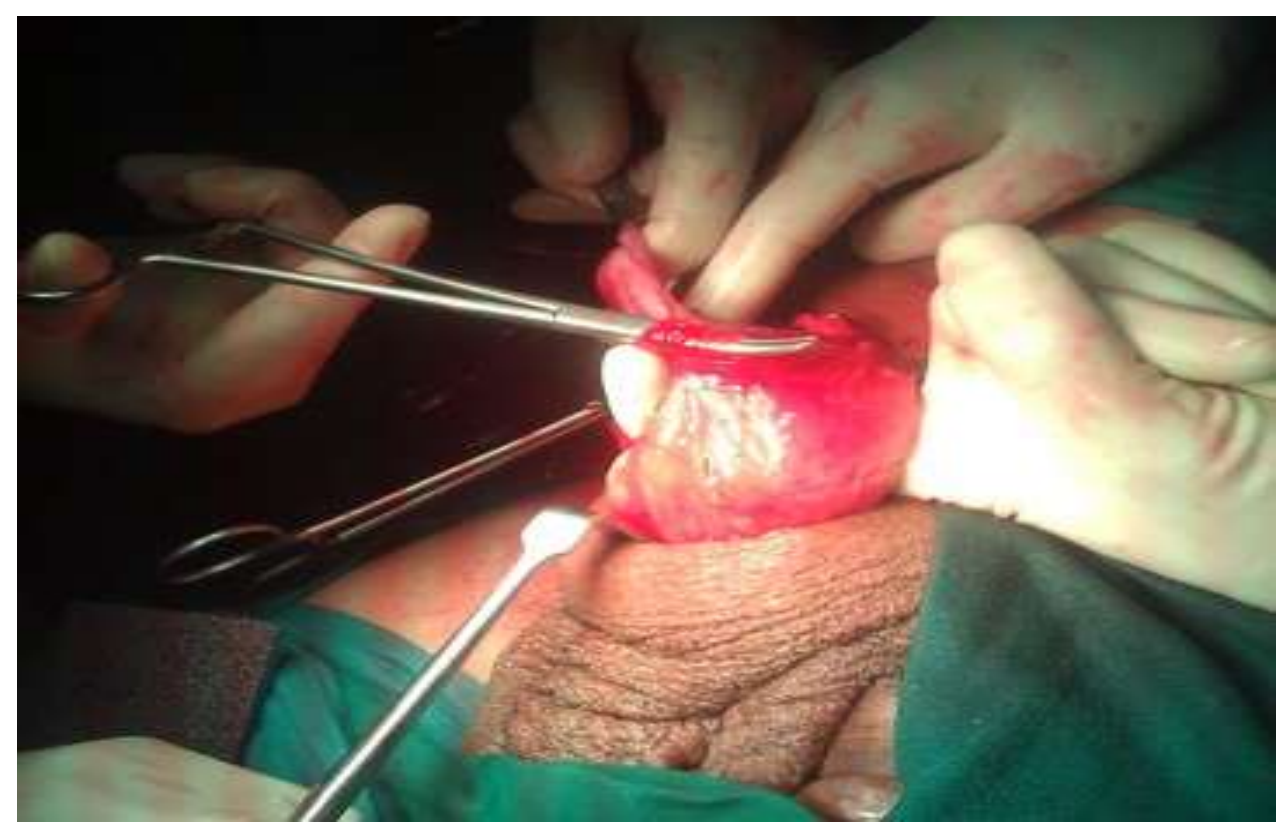

Figure 5: Separating the cord structure from the huge thickened inguinal sac.

During in ICU stay, his initial IAP was between $12-14 \mathrm{mmHg}$ and with moderate ventilator setting. Post-operative blood investigation and blood gases were unremarkable with no evidence of acidosis. However, up to post-operative Day 3, the intra-abdominal pressure was slowly rising to a reading of $28-30 \mathrm{mmHg}$. But, there was no association with organ failure. By POD5 the IAP reduced to $12-14 \mathrm{mmHg}$ and the patient was finally extubated by post-operative day 6 . He was later discharged to main surgical ward on the next day and obtained bowel function after few days with no other major complication. The patient was later discharged home well after one week later. 


\section{Discussion}

Routine hernia surgery becomes anything but routine in a hernia emergency. Incarceration and strangulation are absolute indications for hernia repair. The original data suggesting that all hernias be repaired comes from a series of 439 hernias that quoted the probability of strangulation at 3 months to be $2.8 \%^{3-4}$. This risk seems to be increased by years up to a level whereas the hernia enlarges with time which the risk of strangulation eventually diminished ${ }^{4}$. The standard hernia repair soon becomes difficult in the setting of swelling and tissue distortion together with adhesion occurring within the sac due to patient's manipulation or following traditional treatment such as massage. In addition, the risk of bowel compromise and the need for segmental bowel resection changes the operative approach and choice of method for repair. The morbidity and mortality of hernia repair increases in the setting of emergent hernia which present as obstruction or strangulation.

Giant inguinal hernias such as in this case review can present with several specific problems ${ }^{2}$. The massive size of the hernia can interfere with walking and doing routine work. Penis can be buried inside the scrotum causing urine to dribble over the scrotal skin, which is already congested by lymphatic and venous oedema, causing excoriation, ulceration and secondary infection of the scrotal skin. The ipsilateral spermatic cord will be greatly elongated and obviously prone to torsion. The testis in most of the cases is often atrophic. While these complications already become a troublesome to the patient, obstruction, incarceration and strangulation of the hernial contents further complicates the matter. Complications related to strangulation of the contents in giant inguinal hernias can even be fatal due to high chances in developing bowel ischaemia and perforation.

In patients with giant inguino-scrotal hernias the abdominal viscera is chronically sitted outside the abdominal cavity and often the abdominal cavity has became adapted of being empty. Reduction of the herniated viscera leads to a sudden increase of intra-abdominal and intra-thoracic pressures that can lead to respiratory compromise and eventually multiple organ failure. This is associated with a high mortality rate ${ }^{5,6}$. Several techniques have been described to address this loss of domain including debulking of abdominal contents or enlarging the abdominal cavity. Extensive bowel resections, for example total or hemicolectomy as well as omentectomy and even small bowel resections have been described. Several musculocutaneous flaps have been used and component separation techniques have also been described ${ }^{2,4}$. In this case, none of these techniques were necessary.

Following primary repair, the possibilities of recurrence is high in such giant hernias that were treated by conventional repairs such as in this case. Other possible complication may be related to the remaining redundant scrotal skin. However, considerable shrinkage of the scrotal skin might occur from retraction of the dartos muscle. In addition it may be safer to leave the skin intact as a safety net so that if early postoperative severe respiratory compromise occurs the contents can be temporarily shifted back into the scrotum ${ }^{1,2}$.

As a conclusion, giant inguinal hernias are uncommon but can present a challenging problem to the treating surgeon, as they can lead to potentially fatal complications especially when they presented as obstruction due to the possible development of intra-abdominal hypertension (IAH) and eventually abdominal compartment syndrome (ACS) upon successful reduction of the chronically herniated abdominal contents. Thus the management of giant inguinal hernia poses unique challenges and careful preoperative preparation and close postoperative monitoring is essential to ensure the safety of the patient and to prevent mortality.

\section{References}

[1] Coetzee E, Price C, Boutall A (2011). Simple repair of giant inguinoscrotal hernia. Int J of Sur; 2; $32-35$.

[2] Tahir M, Ahmed F U, Seenu V (2008). Giant inguinoscrotal hernia: case report and management principles. Int J of Surg; 6; 495 497.

[3] Geerard L B, Oosterhuis K J, Peter M.N.Y.H. , et al (1997). Longterm follow-up (12-15 years) of a randomized controlled trial comparing Bassini-Stetten, Shouldice, and high ligation with narrowing of the internal ring for primary inguinal hernia repair. $J$ Am Coll Surg; 185: 352-357.

[4] Inguinal Hernia in the 21st Century: An Evidence-Based Review (2008). Curr Probl Surg; 45: $261-312$.

[5] Hayden P ( 2007). Intra abdominal hypertension and the abdominal compartment syndrome. Curr Anaes \& Crit Care; 18; $311-316$.

[6] Clare B S, Vyas H (2004). Intra-abdominal hypertension and the abdominal compartment syndrome. Curr Paed; 14; 191-196. 